\title{
Desenvolvimento de um curso on-line sobre o tema administração de medicamentos
}

\author{
DEVELOPMENT OF A ON-LINE COURSE ABOUT ADMINISTRATION OF MEDICATION \\ DESARROLLO DE UN CURSO VIA ELECTRÓNICA SOBRE LA ADMINISTRACIÓN DE MEDICAMENTOS
}

\author{
Silvia Helena De Bortoli Cassiani ${ }^{1}$, Flávia Borges da Silva Benfati ${ }^{2}$, Denise Costa Dias ${ }^{3}$, \\ Silvia Helena Zem-Mascarenhas ${ }^{4}$, Maria Lúcia Zanetti ${ }^{5}$
}

\footnotetext{
1 Enfermeira. Professora Associada do Departamento de Enfermagem Geral e Especializada da Escola de Enfermagem de Ribeirão Preto da USP (EERP/USP).

2 Analista de Sistema. Bolsista de Capacitação técnica da Fundação de Amparo à Pesquisa do Estado de São Paulo (FAPESP)

3 Enfermeira. Doutoranda do Programa de Pós-Graduação Área Enfermagem Fundamental da EERP/USP.

4 Enfermeira Doutora em Enfermagem pela EERP/USP

5 Enfermeira. Professora Doutora do Departamento de Enfermagem Geral e Especializada da EERP/USP
}

\author{
RESUMO \\ O estudo descreveu o \\ desenvolvimento de um curso \\ on-line sobre o processo da \\ administração de \\ medicamentos - vias. Para \\ tanto se utilizou o World Wide \\ Web Course Tools (WebCT), \\ que é um software que fornece \\ várias ferramentas a \\ supervisores e acesso a \\ estudantes providos de uma \\ senha a diversos ambientes. \\ O material do curso foi \\ subdivido em oito tópicos \\ ilustrados: administração de \\ medicamentos por via oral, \\ sublingual, via parenteral, \\ intradérmica, subcutânea, \\ intramuscular, endovenosa e \\ aplicação tópica e instilação, \\ além das questões para \\ avaliação de conhecimentos. \\ Uma amostra de alunos de \\ graduação da Escola de \\ Enfermagem de Ribeirão \\ Preto - USP avaliou \\ positivamente o curso. \\ Pretende-se agora inseri-lo \\ em disciplina curricular do \\ ensino de graduação, como \\ um recurso adicional à \\ aprendizagem dos alunos.
}

\section{PALAVRAS-CHAVE}

Educação a distância. Enfermagem.

Vias de administração de medicamentos.

\begin{abstract}
The study describes the development of an on-line course on medication administration. The World Wide Web Course Tools (WebCT) that was used is software that provides various tools for supervisors and access to a number of environments for students who have a password. The course material is presented in a series of websites which are sub-divided into eight illustrated topics concerning medication administration routes, in addition to questions for knowledge assessment. A sample of undergraduate students at the University of São Paulo at Ribeirão Preto College of Nursing has positively evaluated the course. It is now intended to be included as curricular course in the undergraduate program as an additional resource for student learning.
\end{abstract}

\section{KEYWORDS}

Education, distance.

Nursing

Drug administration routes

\section{RESUMEN}

El estudio describe el desarrollo de un curso via internet sobre el proceso de administración de medicamentos - vias. Para tal fin se utilizó el World Wide Web Course Tools (WebCT), que es un software que proporciona varias herramientas a supervisores y acceso a estudiantes, provistos de una clave, a diversos ambientes. El material del curso fue subdividido en ocho tópicos ilustrados: administración de medicamentos por via oral, sub-lingual, via parenteral, intradérmica, subcutánea, intramuscular, endovenosa y aplicación tópica e instilación, contiene además preguntas para la evaluación de conocimientos. Una muestra de alumnos del pre grado de la Escuela de Enfermería de Ribeirão Preto - USP evaluó positivamente el curso. Ahora se pretende incluirlo en la disciplina curricular del pre grado, como un recurso adicional para el aprendizaje de los alumnos.

\section{PALABRAS-CLAVE}

Educación a distancia. Enfermería. Administración de medicamentos 


\section{INTRODUÇÃO}

O processamento racional da informação por meios automáticos, especialmente computadores, caracterizou o surgimento na década de 60 de uma nova ciência denominada Informática, que surgiu da união dos vocábulos "informação" e "automática" (1).

Desde então as mudanças tecnológicas vêm ocorrendo muito rapidamente e tecnologias, apresentadas como a última palavra em termos de pesquisa de ponta, tornam-se ultrapassadas em poucos meses. Decorrente disto, o setor de informática aumenta sua produtividade constantemente e estimula a competitividade de seus produtos no mercado, o que diretamente influencia empresas e sociedade em geral.

Estas mudanças tecnológicas tiveram repercussão na educação, já que a qualidade e a quantidade de recursos humanos bem treinados definem o ritmo e a natureza das transformações sociais e econômicas. Inúmeras possibilidades estão sendo inseridas no ambiente educacional principalmente no que diz respeito à educação a distância e educação continuada. Os limites, embora não plenamente conhecidos, estão influenciando profundamente o trabalho nas escolas e o papel dos docentes.

Na área da saúde não poderia ser diferente e o enfermeiro deve estar apto para utilizar a informática. Ações sistematizadas para treinar pessoal, atualizar conhecimentos, padronizar ações básicas de enfermagem e desenvolver habilidades técnicas específicas, dentre outras, são atividades que promovem uma melhoria na qualidade de assistência de enfermagem. Assim, a informática educativa pode ser utilizada nas atividades de aperfeiçoamento de pessoal e de educação continuada.

Várias tecnologias podem ser empregadas como CD-ROM, vídeo-conferência e a instrução mediada pela internet; todas possibilitando o estudo seguindo o ritmo, a hora e local de preferência do aluno.

Nos EUA, a instrução mediada pela internet (IMI) e recursos tecnológicos como a vídeo-conferência têm tornado possível o oferecimento de cursos de pós-graduação, educação continuada, graduação e outros, no formato parcial ou inteiramente à distância contribuindo especialmente para a forma- ção de um profissional com perfil mais compatível com a cultura tecnológica. ${ }^{(2-6)}$

As razões para a utilização da IMI são várias. A cada dia um número crescente de organizações começa a fazer uso dos métodos de comunicação eletrônica, além disso familiarizar os profissionais de enfermagem no consumo de informações via internet, utilizando-a como forma de comunicação com seus pares, na orientação de pacientes, na coordenação de grupos de auto-ajuda e em sua atualização profisssional, entre outras são algumas razões. A IMI pode auxiliar o aprendizado de forma importante no desenvolvimento de uma ecologia de informações ${ }^{(a)}$ através de trabalhos cooperativos e de socialização de experiências relacionadas a um tema. Assim a internet deve ser vista como um meio de apoiar a indagação, composição, colaboração e comunicação entre alunos e orientadores ${ }^{(7)}$.

Embora a Internet (World Wide Web) seja rica em recursos educacionais que podem promover e elevar a aprendizagem, a rede não é um canal de conteúdo cuidadosamente controlado, ao contrário, caracteriza-se pela diversidade com materiais de boa qualidade $\mathrm{e}$ de qualidade duvidosa. Assim, existe a necessidade de orientar os alunos para a utilização mais efetiva das informações e na realização de buscas mais eficientes ${ }^{(7)}$. Ensinar através da Web é uma experiência nova para muitos professores e requer uma reconceitualização de suas regras ${ }^{(8)}$.

A constatação desta situação tem trazido desafios pois conhecer as diversas tecnologias da informação e comunicação e saber utilizá-las para vencer os desafios impostos em cada realidade educacional específica, estar aberto a mudanças, procurar entendê-las e ter disposição de reciclar-se e atuar em cenários diferentes são atitudes condizentes com os tempos modernos ${ }^{(9)}$.

Todavia a maior vantagem é o fato de que o uso dessas tecnologias propicia ao aluno o tempo necessário para que ele progrida, dominando o conteúdo a partir de seu ritmo de aprendizagem,delegando-o maior responsabilidade por sua aprendizagem, de acordo com suas necessidades, capacidades e disponibilidade de tempo. É necessário, entretanto, a organização de uma infra-estrutura educacional que favoreça a efetividade do esforço do aluno.
Desenvolvimento de um curso on-line sobre o tema administração de medicamentos

\footnotetext{
(a) Nardi \& O'Day (1999) utilizam uma metáfora para a tecnologia, referindo-se a ela como parte de um sistema ecológico de informações, envolvido por uma densa rede de relações de ambientes locais. Na ecologia de informações o foco central deve ser as atividades humanas que utilizam a tecnologia. Ecologia contém a noção de evolução contínua, e a idéia de comunidade não coloca a mesma ênfase na mudança. Um sistema ecológico de informações leva tempo para crescer, da mesma forma que uma floresta, e começa com o nosso próprio esforço no envolvimento e uso responsável da tecnologia.
} 
Silvia Helena De B.Cassiani Flávia Borges da S.Benfati Denise Costa Dias Silvia H. Zem-Mascarenhas Maria Lúcia Zanetti
Nesse contexto a educação a distância se coloca como um processo educativo em que a aprendizagem é realizada com uma separação física entre alunos e professores. $\mathrm{O}$ aprendizado e a comunicação acontecem por meio de recursos tecnológicos que ultrapassam a exposição oral, o supervisor ou professor passam a assumir responsabilidades adicionais, ou seja, começa-se a repassar o conteúdo disciplinar via cabo, através de programas instrucionais e na forma on-line. Essas vantagens são especialmente válidas quando refletimos os benefícios para os programas de educação continuada nas instituições de saúde.

Os autores desse texto reconhecem a importância da utilização da tecnologia educacional na enfermagem, já desenvolveram materiais instrucionais - CD-ROM com o propósito de inovar o ensino, entretanto, nada tinham ainda desenvolvido que integrasse a Internet ao ensino. Aliado a essa motivação encontra-se também a curiosidade e o interesse que o uso da Internet tem entre o grupo de alunos e sua pouca utilização como estratégia educativa, o que torna esse projeto um dos pioneiros no ensino de enfermagem.

Considerando a experiência com a temática Administração de Medicamentos no ensino de graduação em enfermagem e nas pesquisas optou-se por abordar esse conteúdo num programa de ensino a distância.Essa experiência, embora inicial, mas já avaliada por um grupo de alunos pode estimular novos estudos para a implantação dessa tecnologia no ensino de enfermagem.

\section{OBJETIVOS}

O estudo teve por objetivos:

- desenvolver um curso on-line sobre o processo de administração de medicamentos-vias, para ser utilizado no ensino de graduação em enfermagem, utilizando-se a ferramenta do WebCT;

- realizar a avaliação preliminar deste curso com uma amostra de alunos de graduação em enfermagem.

\section{METODOLOGIA}

Trata-se de um estudo descritivo que relata o processo de desenvolvimento de um curso on-line sobre o processo da administração de medicamento - vias, ou seja, seu planejamento, desenvolvimento, avaliação preliminar e revisão. $\mathrm{Na}$ fase de avaliação contou-se com a participação de uma amostra de alunos de graduação em enfermagem da Escola de Enfermagem de Ribeirão PretoUSP, que participaram voluntariamente e assinaram termo de consentimento informado em duas vias, respeitando os aspectos éticos preconizados pela resolução 196/96. Preservou-se a livre participação e a desistência a qualquer momento, sem que isso incorresse em qualquer prejuízo para o aluno. Essa amostra de conveniência incluiu 28 alunos do $2^{\circ}$ e $3^{\circ}$ ano do curso de graduação que demonstraram interesse pelo tema e pela estratégia de ensino-aprendizagem e responderam a um convite aberto a todos os alunos.

Para a avaliação preliminar do curso foi desenvolvido um instrumento baseado no modelo de PRICE (1991) contendo três categorias. A primeira categoria se referia a adequação instrucional e foram avaliados os seguintes itens: apresentação do menu de entrada, conteúdo, tamanho do conteúdo nos tópicos, clareza do texto, seqüência instrucional dos tópicos, apresentação dos procedimentos técnicos dos tópicos, oportunidade de instrução individual e motivação. A segunda categoria referiu-se a adequação estética e analisou-se o uso do espaço da tela, qualidade das figuras e apresentação visual das questões. E a terceira categoria avaliou a adequação do curso via Internet através dos seguintes itens: facilidade de execução do programa, entrada no programa, apresentação das instruções de uso, questões para avaliação de aprendizagem e saída do programa.

\section{DESENVOLVIMENTO DO CURSO}

A programação do curso seguiu, portanto, os seguintes estágios: planejamento, desenvolvimento, avaliação preliminar e revisão. No processo de planejamento foram determinadas as necessidades educativas, analisados o grupo de alunos (a audiência), estabelecidas metas e avaliada a infra-estrutura da instituição para reconhecer a viabilidade ou não do projeto.

Percebeu-se que existia a necessidade e motivação para a utilização de tecnologias da informação e comunicação na educação 
de enfermagem, que a instituição oferecia condições para o desenvolvimento do curso on-line e que havia interesse nos tópicos programados sobre administração de medicamentos. Assim o projeto foi considerado viável.

O desenvolvimento incluiu a criação do conteúdo, a revisão de material existente sobre o tema, a organização e desenvolvimento do conteúdo e a seleção/desenvolvimento de conteúdo. Depois de selecionado o conteúdo com as devidas ilustrações a partir da experiência dos autores, este foi inserido no ambiente WebCT, subdividido em tópicos ilustrados com fotos e figuras além de conter textos explicativos do assunto.

O WebCT é um pacote educacional que fornece a supervisores, professores e alunos providos de uma senha, acesso a diversos ambientes educacionais e instrumentos administrativos incluindo e-mail, calendário, boletim, chat, notas do curso, exercícios, dentre outros. Lançado comercialmente em 1997 pela empresa WebCT, hoje com sede em Boston - USA, foi desenvolvido originalmente na Universidade de British Columbia no Canadá, em 1995, com o objetivo de atender às necessidades do mercado acadêmico. Desde então se tornou o sistema de gerenciamento de cursos on-line bastante popular. Várias instituições educacionais utilizam-no para ministrar cursos (http://www.people computacao.com.br/ webct/comerc.htm).

Uma vez instalado em um servidor, o WebCT pode abrigar inúmeros cursos. Pode ser usado para elaboração de cursos inteiramente on-line ou como ferramenta de apoio a cursos presenciais, suplementando as tradicionais classes face-a-face. A escolha deste software deveu-se ao fato de este encontrarse disponível na instituição e constituir um instrumento apropriado para a educação a distância, como já mencionado.

O WebCT conta com várias ferramentas que podem ser inseridas no curso, entre elas o Sumário, que exibe o conteúdo em tópicos; clicando-se em cada item o aluno verá o conteúdo a ele relacionado, 6 salas de batepapo que permitem troca de informações entre os alunos e também com o professor, podendo ser usadas para aulas on-line e esclarecimento de dúvidas em horários pré-programados, Revisão, onde o aluno escolhe os tópicos do curso e o programa monta uma página apenas com os tópicos selecionados, para visualização ou impressão, Agenda local onde estão os avisos importantes dados pelo supervisor, tais como datas de provas, trabalhos e aulas especiais, Avaliações que exibem as notas de provas e trabalhos sendo que cada aprendiz tem acesso somente à sua nota e gabaritos, onde é possível disponibilizar resultados e comentários de provas preparados pelos docentes.

As questões para avaliar o conhecimento dos alunos depois de respondidas podem ser enviadas para o endereço eletrônico do professor como estratégia de avaliação, ou mesmo discutidas em chat ou fórum com o professor e outros alunos cadastrados no curso. Para cada tópico há notas, objetivos, exercícios, podendo ser introduzidos links para outros recursos da web. O programa e o calendário direcionam o aluno através do curso semanalmente.

A Figura 1 apresenta uma página com a tela inicial do curso denominado de intervenções de enfermagem relacionadas ao processo de administração de medicamentos Vias.

Portanto a partir do sumário encontramse os seguintes tópicos de estudo: administração de medicamentos por via oral e por via sublingual; por via parenteral: via intradérmica; via subcutânea, via intramuscular; aplicação tópica e instilação e administração de medicamentos por via endovenosa e questões para avaliar o conhecimento, apresentados na Figura 2. Vale destacar que somente os alunos cadastrados no curso pelo professor podem acessá-lo.

A Figura 3 apresenta uma tela com o conteúdo do curso propriamento dito
Desenvolvimento de um curso on-line sobre o tema administração de medicamentos 
Silvia Helena De B.Cassiani Flávia Borges da S.Benfati Denise Costa Dias Silvia H. Zem-Mascarenhas Maria Lúcia Zanetti

\section{ERG 218: CUIDADOS FUNDAMENTAIS DE ENFERMAGEM AO HOMEM ADULTO.}

\section{Módulo 1 -Intervenções de enfermagem relacionadas ao processo de administração de medicamentos - Vias}

Objetivos: Identificar as vantagens e desvantagens da administração de medicamentos nas várias vias. Descrever os procedimentos na Administração de Medicamentos. Listar os cuidados a serem tomados. Citar o material necessário para se administrar a medicação nas vias oral, intradérmica, subcutânea ,intramuscular e endovenosa.

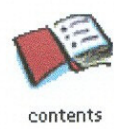

Sumário

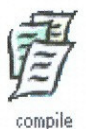

Revisão da Matéria

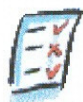

quiz

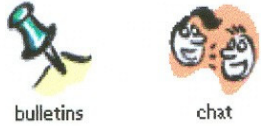

Fórum

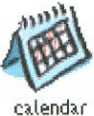

Agenda

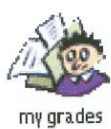

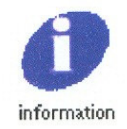

Ajuda

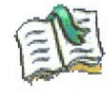

resume session

Último item
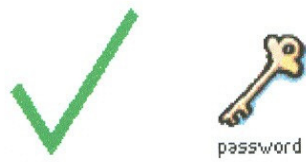

Senhas

Teste seus conhecimentos

Esta página foi acessada 00093 vezes.

Figura 1: Tela inicial do Web CT.

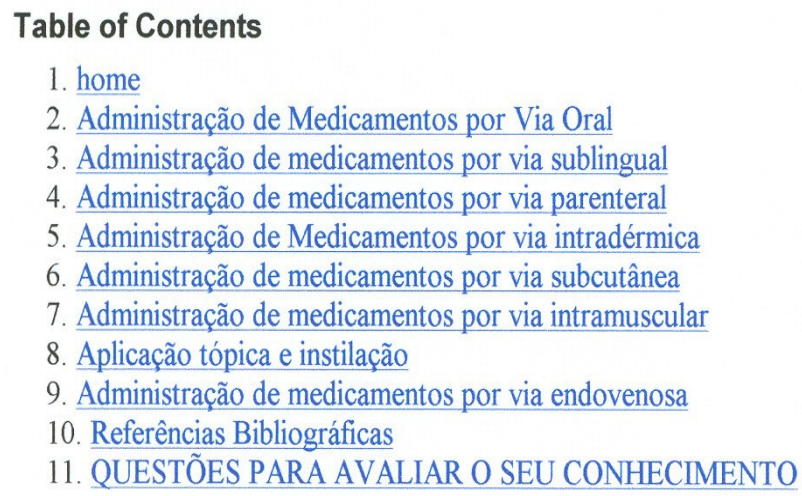

Esta página foi visitada 00078 vezes

Rev Esc Enferm USP 2003; 37(3): 44-53
Figura 2- Sumário do Curso 


\section{ADMINISTRAÇÃO DE MEDICAMENTOS POR VIA SUBLINGUAL}



( Fonte: MILLER, D. Medication Administration. Springhouse Corporation, 506p, 2000)

Medicamentos sublinguais seguem o mesmo procedimento empregado para aqueles de via oral, exceto a colocação do comprimido sob a língua. Para tanto solicita-se que o paciente abra a boca e repouse a língua no palato; a seguir, coloca-se o medicamente sob a língua, usando uma gaze para segurá-lo e o paciente permanecerá com o medicamento, nesse local, até sua completa absorção. 0 paciente não deve conversar nem ingerir líquidos ou alimentos durante esse período.

Se a apresentação for em gotas, as mesmas devem ser pingadas diretamente na boca do paciente, sob a língua.

Os medicamentos por via sublingual promovem uma rápida absorção da droga em curto espaço de tempo, além de se dissolverem rapidamente, deixando pouco resíduo na boca.

Como a absorção por via sublingual é importante em muitos casos, por exemplo, na administração de nitroglicerina. Como a drenagem venosa da boca, desemboca na veia cava superior, os medicamentos estão protegidos do metabolismo de primeira passagem pelo fígado, o que é vantajoso.

\section{Esta página foi visitada 00035 vezes}

Figura 3 - Tela do módulo Administração de Medicamentos pela via sublingual

Para avaliar o aprendizado de uma classe em aulas presenciais, utilizamos uma variedade de técnicas como questões, comentários, monitoramento das expressões de linguagem corporal e facial e ajustamento de estratégias como diminuir o ritmo, rever o material como resposta às questões, confusões ou mau entendimento ou aumentar o ritmo quando as atitudes dos estudantes superam as expectativas. Entretanto, quando o ensino é a distância há desafios como não ter mais: uma classe tradicional, um grupo homogêneo de alunos, "feedback" face a face durante a aula, controle total, oportunidades convenientes de conversar com os alunos individualmente. Dessa forma pode ser mais útil, não somente avaliar os estudantes através de testes e exercícios, mas usar uma abordagem mais informal que determine a clareza do conteúdo do curso, a efetividade do ensino, como o curso pode ser melhorado ou aperfeiçoado etc.

Nesse curso a avaliação de conhecimentos foi baseada nas respostas às questões de avaliação embora outras estratégias possam
Desenvolvimento de um curso on-line sobre o

tema administração de medicamentos 
Silvia Helena De B.Cassiani Flávia Borges da S.Benfati Denise Costa Dias Silvia H. Zem-Mascarenhas Maria Lúcia Zanetti ser pensadas como número de vezes que o aluno acessou o curso, número de participações nos fóruns e chats e contribuições apresentadas nesses momentos.

\section{Avaliação preliminar do curso on-line sobre administração de medicamentos}

Após a criação do curso e disponibilização do mesmo na Internet efetuamos uma avaliação preliminar com uma amostra aleatória de 28 alunos do $2^{\circ}$ e $3^{\circ}$ ano do curso de graduação em enfermagem da Escola de Enfermagem de Ribeirão Preto -
USP, visando a revisão e aprimoramento do curso antes de oferece-lo de forma efetiva. Há planejamento para inseri-lo no conteúdo de uma disciplina de caráter optativo sobre administração de medicamentos. Os alunos após estudarem através dos tópicos apresentados responderam a um instrumento de avaliação.

A figura 4 ilustra a opinião dos alunos sobre a adequação instrucional. Pode ser observado que a maioria dos avaliadores considerou excelente todos itens, com exceção do item tamanho dos tópicos.

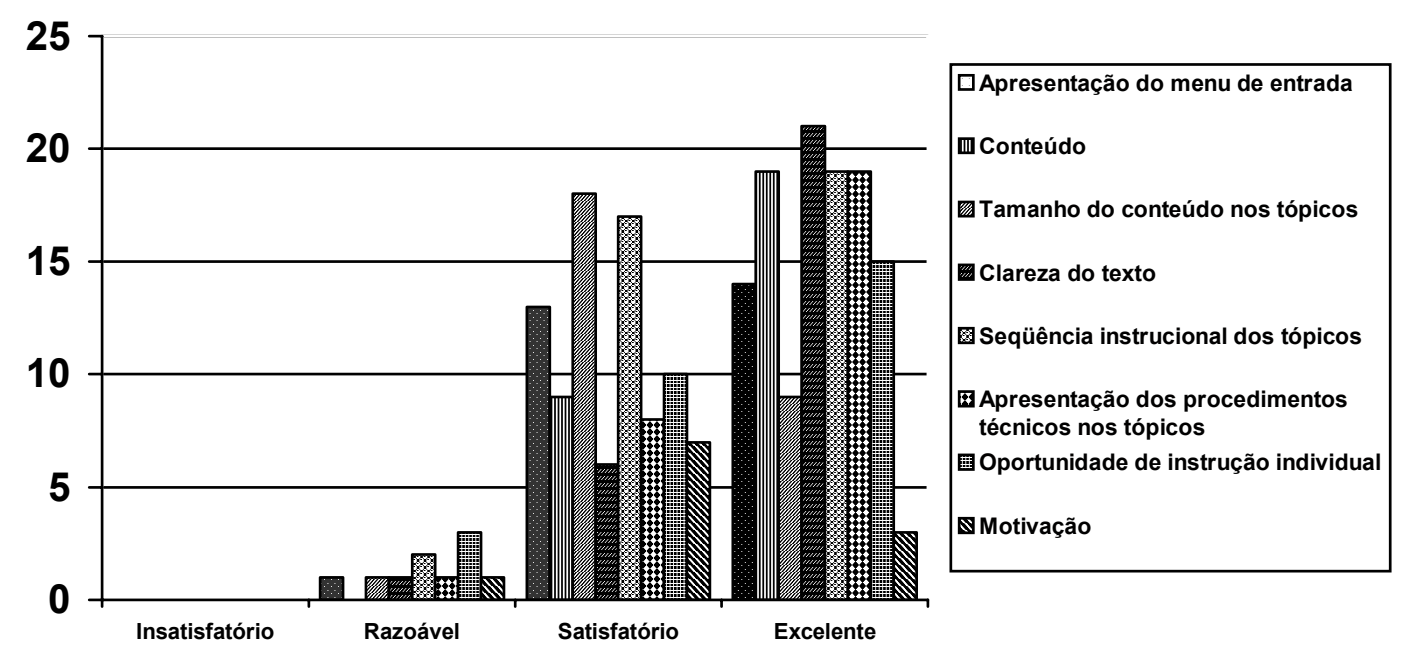

Figura 4 - Respostas dos alunos de graduação em Enfermagem sobre a adequação instrucional do curso. Ribeirão Preto (SP, 2000)

Com relação à adequação instrucional os alunos citaram: o conteúdo muito extenso, a necessidade da abordagem de dificuldades encontradas como fotos e utilização de alguns termos em inglês.

Ressalta-se que um curso WebCT bem planejado deve identificar claramente as atividades de aprendizagem e os resultados esperados e deve ser logicamente organizado, visualmente apelativo e facilmente acessado e navegado. É realmente muito mais fácil avaliar um curso, tal como os alunos realizaram, quando se conhece uma variedade deles, que permite inclusive que comparações sejam feitas. Vale destacar que o WebCT utiliza todos os termos em inglês, que é uma desvantagem e que justifica o relato dos alunos. Sobre o conteúdo considerado extenso, esse será objeto de redução.

A Figura 5, ilustra a opinião dos alunos sobre a adequação estética do curso on-line sobre administração de medicamentos no ambiente WebCT. 




\section{Uso do espaço da tela \\ 国 Qualidade das figuras}

図 Apresentação visual das questões
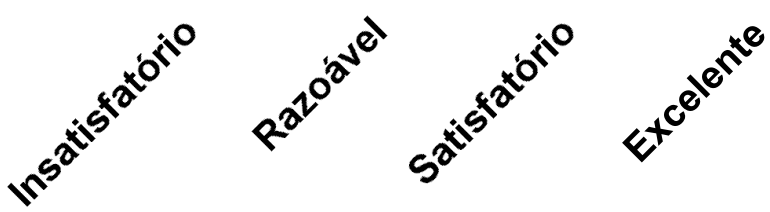

Figura 5 - Resposta dos alunos de graduação em enfermagem sobre a adequação estética do curso. Ribeirão Preto (SP, 2000)

A qualidade das figuras foi considerada excelente pela maioria dos avaliadores, já o uso do espaço da tela e apresentação visual das questões foram considerados satisfatórios. Um avaliador considerou o item uso do espaço da tela razoável e quatro avaliadores consideraram o item qualidade das figuras como razoável.

Um dos avaliadores apresentou o seguinte comentário: aumentar o tamanho do quadro do canto superior esquerdo que indica o botão para passar à página seguinte ou anterior. Este comentário referia-se a parte estrutural do programa e não há como ser modificado.

Quanto à qualidade das figuras estas serão, também, objeto de avaliação por esses autores.

A Figura 6 indica as respostas dos alunos sobre a adequação do curso via Internet.

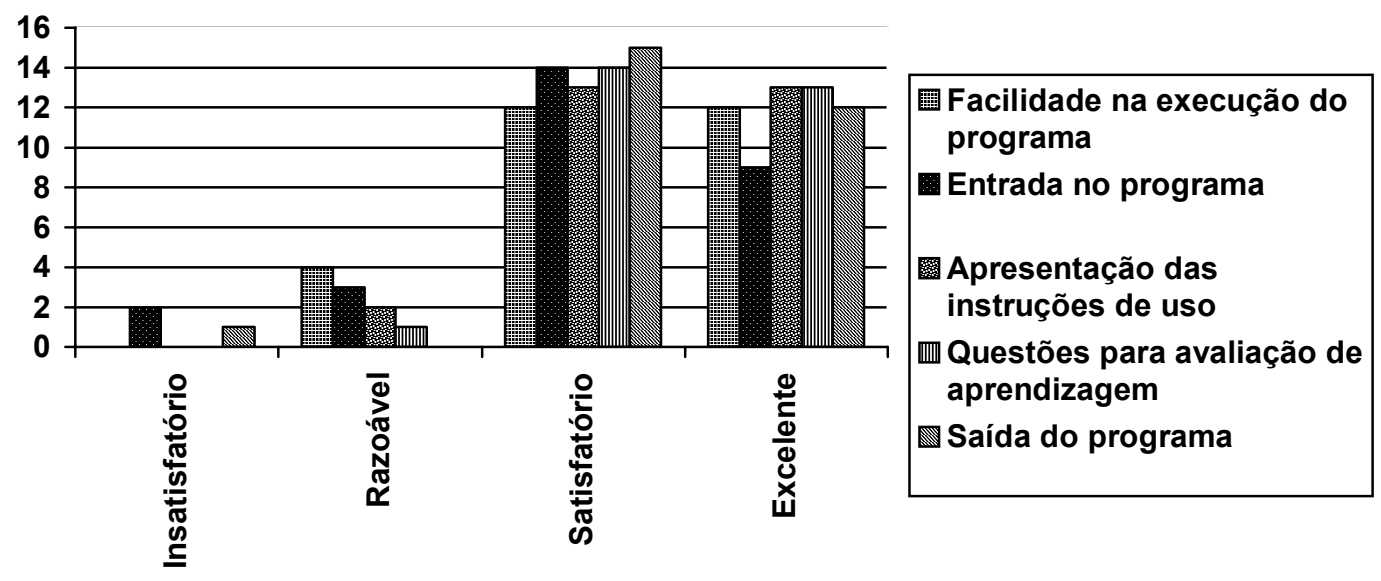

Figura 6 - Respostas dos alunos de graduação em enfermagem sobre adequação do curso Ribeirão Preto (SP, 2000) 
Silvia Helena De B.Cassiani Flávia Borges da S.Benfati Denise Costa Dias Silvia H. Zem-Mascarenhas Maria Lúcia Zanetti
A facilidade na execução do programa foi considerada satisfatória e excelente por 12 avaliadores e razoável por 04, conforme o gráfico 3. Já os itens entrada no programa, apresentação das instruções de uso, questões para avaliação da aprendizagem e saída do programa foram consideradas satisfatórios pela maioria dos avaliadores e apenas 02 consideram como insatisfatória a entrada do programa.

Obtivemos os seguintes comentários gerais:

Muito interessante esse curso via Internet, principalmente por abordar o tema de forma clara. Assim podendo ser usado por alunos em fase de aprendizado e também por profissionais em caso de dúvida;

Acho que é uma ótima forma de aprendizado e facilita o acesso ao material didático pelo aluno;

Muito interessante, porém penso que o conteúdo poderia ser mais breve.

Cursos via on-line tem vantagens como: a flexibilidade, a melhoria das habilidades no computador, aumento no acesso da informação e a aprendizagem independente, enquanto que as desvantagens são as dificuldades com a tecnologia, a diminuição no contato com os pares e docentes e o aumento na demanda do tempo ${ }^{(10)}$. Nessa fase os alunos que revisaram o curso viram pontos positivos, embora alegassem que o conteúdo fosse extenso.

Esse ponto abordado pelos alunos levou à revisão do material buscando diminuir o conteúdo do curso, sem alterar o número de tópicos. A próxima fase será a de divulgar o curso realizando-o sob o formato de disciplina optativa do curso de graduação.

\section{CONCLUSÕES}

A educação a distância não substitui a educação presencial, mas pode ser uma segunda opção para aqueles que por algum motivo não podem participar da educação presencial ou mesmo como uma ferramenta de apoio ao ensino. Geralmente o "aluno distante" é mais velho, trabalha, tem família e precisa coordenar as diferentes áreas de sua vida; tem uma variedade de razões para fazer os cursos: ou mandatório pela instituição ou pela necessidade de algum conhecimento adicional.

Essa modalidade de ensino, como outra qualquer apresenta vantagens e desvantagens. Uma vantagem é o envolvimento dos alunos através de cursos on-line ficam ativamente envolvidos em estudar (leitura, visitas a sites e comunicação eletrônica) enquanto que os alunos no ensino tradicional sentamse passivamente numa sala ${ }^{(11)}$.

Proporciona também oportunidade de rico intercâmbio de informações e idéias no qual é importante a participação ativa dos alunos, que devem ser orientados não apenas para a assimilação e reprodução, mas, na interação entre alunos e professor, a problematizar, analisar, refletir e discutir. Uma característica que distingue o ensino on-line do ensino presencial, é o fato de que os alunos têm tempo para elaborarem e refletirem sobre suas contribuições antes de disponibilizá-las para o grupo, diferindo de contribuições orais realizadas em salas de aula onde falam o que está na "ponta da língua", sem tempo para maior reflexão ${ }^{(12)}$.

Como desvantagem aparece a dificuldade para a relação interpessoal. Geralmente o aluno está isolado e os fatores motivacionais provenientes do contato ou competição com outros estão ausentes, falta ainda o apoio do professor que poderia lhe dar um suporte imediato ou motivá-lo, dando atenção às suas necessidades. Na educação a distância, a tecnologia é tipicamente o suporte através do qual a informação e comunicação fluem.

Uma limitação que precisa ser considerada é o custo, uma vez que é importante considerar os recursos necessários como equipamento, manutenção, infraestrutura, produção e pessoal. Desta forma é necessário investimento em infra-estrutura da instituição provedora.

Todavia, a utilização deste tipo de tecnologia é importante uma vez que tanto o aluno quanto o profissional precisam estar sempre atualizados e nem sempre há disponibilidade para atualizar os conhecimentos na forma presencial. Para o futuro pode-se prever uma generalização deste tipo de estratégia nos mais diversos campos, um aumento da eficiência da utilização das diferentes tecnologias e oferta contínua de utilização de estratégias típicas de EAD em atividades presenciais.

As atividades desenvolvidas para a elaboração desse curso mostraram além de suas dificuldades próprias para o desenvolvimento de uma tecnologia educacional ainda inovadora nos nossos meios, a possibilidade 
de seu uso para a educação continuada e a distância nas nossas instituições. O investimento feito é muitas vezes bem abaixo dos benefícios advindos com a aprendizagem dos alunos/ profissionais. Resta, todavia, um olhar mais atento dos profissionais e das instituições para a utilização da tecnologia usando a internet.

Este estudo encontra-se na sua primeira fase, qual seja o desenvolvimento do curso e avaliação através de um grupo de alunos que embora considerassem excelentes muitos dos itens avaliados destacaram pontos que merecem ser analisados quando da versão definitiva. Apontamos, entretanto, sua importância pela inovação e possibilidade de que a partir desse estudo outros venham a desenvolver programas usando essa ferramenta. É seu emprego efetivo o desafio seguinte na educação em enfermagem.

\section{REFERÊNCIAS}

(1) Youssef NA, Fernandez VP. Informática e sociedade. São Paulo: Ática; 1985.

(2) Association of American Colleges of Nursing. The essentials of baccalaureate education for professional nursing practice. Washington (DC); 1998.

(3) Sternberger C, Meyer L. Intermedia Assistent Instruction: Authoring with learning guidelines. Comput Nurs 2001; 19:69-74

(4) Rosenlund CH, Damask-Bembenek B. Assessing the effectiveness of an on-line program. Nurse Educ 1999; 24:5-6.

(5) Carlton KH, Ryan ME, Siktberg LL. Designing courses for the internet: a conceptual approach. Nurse Educator, 1998; 23:45-50.

(6) Sery - Ble OR, Taffe ER, Clarke AW, Dorman T. Use of and satisfaction with a browser based nurse teaching tool in a surgical intensive care unit. Comput Nurs 2001; 19: 82-6

(7) Nardi, BA; O'Day, V. Information ecologies: using technology with heart. Cambridge: MIT Press, 1999.
(8) Halstead JA, Coudret NA. Implementing Webbased instruction in a school of nursing: implications for faculty and students. J Prof Nurs 2000; 16:273-81.

(9) Carvalho GMG, Botelho FVU. Educação a distância: um estudo sobre expectativas dos alunos em relação ao uso do meio impresso e eletrônico, 2001. http://www.intelecto.nt/ ead textos/glaucia1.htm> (05mar.2001).

(10) Gore PJW. Developing and teaching on-line courses in geology at the two year college level in Georgia. Comput Geosci 2000; 26:641-6.

(11) Harassin LM. Learning Networks- a field guide to teaching and learning on-line. Boston: The MIT Press; 1995.

(12) Price RV. Computer-aid instruction: a guide for authors. Pacific Grove: Brooks/Cole Publishing;1999.
Desenvolvimento de um curso on-line sobre $o$ tema administração de medicamentos 